\title{
Coherent Radiation Generation and Amplification in Erbium Doped Systems
}

\author{
Sterian Andreea Rodica \\ University Politehnica of Bucharest, \\ Romania
}

\section{Introduction}

The erbium doped fiber systems results into important advantages for information processing and transmission like: possibility of easy integration, highly efficiency and gain, immunity to crosstalk, low noise and high saturation output power (Agrawal, 1995 \& 1997; Desurvire, 1995; Sterian, 2006).

During the last years, they have been published many studies carrying out the improving and optimization of the coherent optical systems by "computer experiments". Based on some computational models known in the literature, this chapter proposes to present the main author's results obtained by numerical simulation concerning some coherent optical amplifier and laser systems.

Will be presented firstly the computational model which govern the amplification regime of an uniform doped optical fiber under the form of a system of the nonlinear transport coupled equations, respectively for the signal and for the pumping. This system was used for numerical simulation of the amplification phenomena by a Runge - Kutta type method (Agrawal, 1995; Sterian, 2006; Press et al., 1992).

The study continues with the computational model presentation used for numerical analyses of the laser system doped with $\mathrm{Er}^{3+}$ ions, both of the crystal type and of the optical fiber laser type (Pollnau et al., 1996; Maciuc et al., 2001; Sterian \& Maciuc, 2003).

The main problems studied by numerical simulation, using these models known in literature are: the amplification, the laser efficiency and threshold for different optical pumping wavelengths, the dependence of the output optical power on the levels life time, the influence of the host materials on the output power and the time dependent phenomena, stability and nonchaotic regime of operation (Maciuc et al., 2001; Pollnau et al., 1994).

We realized the numerical simulation of the erbium doped fiber amplifier concerning the functional and constructive parameters. It was demonstrated that for the $\mathrm{Er}^{3+}$ doped fiber laser, the optimum operation condition are obtained for $\lambda=791 \mathrm{~nm}$, when the upper laser level is directly pumped but in the presence of the "colaser" process which improves two times the laser efficiency for $\lambda=3 \mu \mathrm{m}$.

$\mathrm{The} \mathrm{Er}^{3+}$ laser system functionment and optimization was studied for different host material, using 3D numerical simulation to take into account the characteristic parameters variations in the range of values resulting from experience; the material selection recommends as efficient materials LiY $\mathrm{F}_{8}$ and $\mathrm{BaY} \mathrm{F}_{8}$. 
We also put into evidence the existence of a strong interdependence between active medium parameters having important role in the designing of the erbium laser systems with given functional parameters.

We demonstrated the stable, nonchaotic operation of the analyzed laser systems and the modulation performance of them using the communications theory methods.

For the erbium doped fiber laser we explained the complex dynamics of this type of device by simulating the time dependence of the output power correlated with the corresponding changes in the populations of the implied levels.

Another author's numerical simulations refers to nonlinear effects in optical fibers systems (Sanchez et al., 1995; Ninulescu \& Sterian, 2005; Ninulescu et al., 2006;). Self - pulsing and chaotic dynamics are studied numerically in the rate equations approximation, based on the ion - pair formation phenomena (Sanchez et al., 1993; Sterian \& Ninulescu, 2005; Press et al., 1992).

The developed numerical models concerning the characterization and operation of the EDFA systems and also of the laser systems, both of the "crystal type" or "fiber type" realized in $\mathrm{Er}^{3+}$ doped media and the obtained results are consistent with the existing data in the literature.

That was possible due to the valences of the computer experiment method which permits a complex study taking into account parameters intercorrelations by simulating experimental conditions, as have been shown.

The used fourth order Runge - Kutta method for the numerical simulation demonstrates the importance of the "computer experiments" in the designing, improving and optimization of these coherent optical systems for information processing and transmission (Stefanescu et al., 2000, 2002, 2005; Sterian, 2002; Sterian, 2007).

Some new feature of the computer modeled systems and the existence of new situations have been put into evidence, for designers utility in different applications (Petrescu, 2007; Sterian, 2008). Our results are important also for the optimization of the functioning conditions of this kind of devices.

\section{Fiber amplifier}

\subsection{Transport equations for signal and pumping}

Let us consider an optical fiber uniformly doped, the concentration of the erbium ions being $N_{0}$. The pumping is done with a laser radiation having $\lambda_{p}$ wavelength and the pumping power $P_{p}$, the absorption cross - section being $\sigma_{p}^{a}$. The population densities of the atoms on each of the three levels involved in laser process are: $N_{1}(t, z), N_{2}(t, z)$ respectively $N_{3}(t, z)$ which verify the equations:

$$
\begin{gathered}
N_{3}(t, z) \cong 0 \\
N_{1}(t, z)+N_{2}(t, z)=N_{0} .
\end{gathered}
$$

The necessary condition for radiation amplification in this kind of systems is as in the laser case the population inversion.

In the next presentation we refer to the energy levels diagram presented in figure 1 where: $\sigma_{s}^{a}$ is the absorption cross-section for the signal; $\sigma_{s}^{e}$ is the stimulated emission cross-section 
corresponding to the signal; $\sigma_{p}^{a}$ is the absorption cross-section for the pumping radiation and $\tau$ is the relaxation time by spontaneous emission.

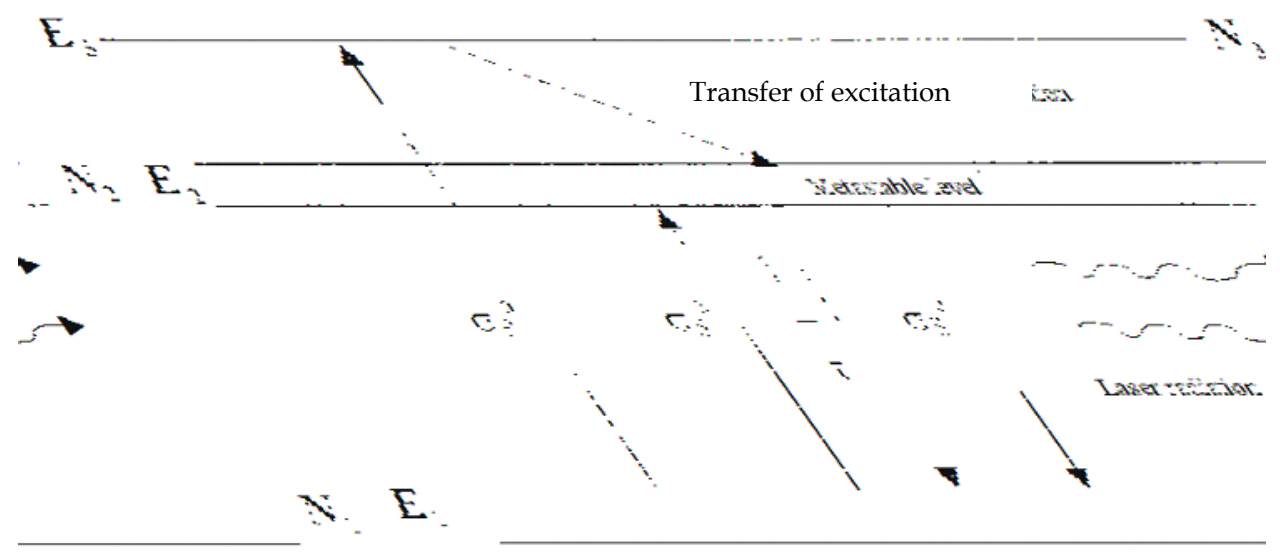

Fig. 1. The diagram of the energy levels involved in radiation amplification

For this system of energy levels on can write three rate equations: one for the population of the $E_{2}$ level and two transport equations for the fluxes of the signal and pumping. These rate equations are respectively (Agrawal, 1995):

$$
\begin{gathered}
\frac{\partial}{\partial t} N_{2}(t, z)=\frac{\sigma_{p}^{a} N_{1}(t, z) \cdot I_{p}(t, z)}{h v_{p}}+\frac{\sigma_{s}^{a} N_{1}(t, z) \cdot I_{s}(t, z)}{h v_{s}}-\frac{N_{2}}{\tau}-\frac{\sigma_{s}^{e} N_{2}(t, z) \cdot I_{s}(t, z)}{h v_{s}} ; \\
\frac{1}{c} \cdot \frac{\partial}{\partial t} I_{p}(t, z)=-\frac{\partial}{\partial z} I_{p}(t, z)-\sigma_{p}^{a} \cdot N_{1}(t, z) \cdot I_{p}(t, z) ; \\
\frac{1}{c} \cdot \frac{\partial}{\partial t} I_{s}(t, z)=-\frac{\partial}{\partial z} I_{s}(t, z)+\sigma_{s}^{e} \cdot N_{2}(t, z) \cdot I_{s}(t, z)-\sigma_{s}^{a} \cdot N_{1}(t, z) \cdot I_{s}(t, z) ;
\end{gathered}
$$

where:

$W_{p}=\frac{\sigma_{p}^{a} \cdot I_{p}(t, z)}{h v_{p}}$ is the absorption rate for the pumping; $W_{s}^{a}=\frac{\sigma_{s}^{a} \cdot I_{s}(t, z)}{h v_{s}}$ - is the absorption rate for the signal; $W_{s}^{e}=\frac{\sigma_{s}^{e} \cdot I_{s}(t, z)}{h v_{s}}$ - is the stimulated emission rate; $\frac{1}{\tau}$ - is the spontaneous emission rate; $\sigma_{p}^{a} \cdot N_{1}(t, z)$ - is the rate of pumping diminishing by absorption; $\sigma_{s}^{e} \cdot N_{2}(t, z)$ - rising rate of the signal by stimulated emission and $\sigma_{s}^{a} \cdot N_{1}(t, z)$ - is the rate of signal diminishing by absorption. (It admit that $W_{s}^{a}=W_{s}^{e}=W_{p}$ ).

In the same time the initial condition are:

$$
I_{p}(t, 0)=I_{p}(t)
$$




$$
I_{s}(t, 0)=I_{s}(t) \text {. }
$$

If the next conditions are fulfilled:

$$
\begin{gathered}
\frac{\partial}{\partial t} N_{2}(t, z)=0, \\
\frac{\partial}{\partial t} I_{p}(t, 0)=\frac{\partial}{\partial t} I_{p}(t, z)=0, \\
\frac{\partial}{\partial t} I_{s}(t, 0)=\frac{\partial}{\partial t} I_{s}(t, z)=0,
\end{gathered}
$$

one obtain the steady state equations:

$$
\begin{gathered}
\frac{\sigma_{p}^{a} N_{1}(t, z) \cdot I_{p}(t, z)}{h v_{p}}+\frac{\sigma_{s}^{a} N_{1}(t, z) \cdot I_{s}(t, z)}{h v_{s}}-\frac{N_{2}}{\tau}-\frac{\sigma_{s}^{e} N_{2}(t, z) \cdot I_{s}(t, z)}{h v_{s}}=0, \\
\frac{\partial}{\partial z} I_{p}(t, z)=-\sigma_{p}^{a} \cdot N_{1}(t, z) \cdot I_{p}(t, z), \\
\frac{\partial}{\partial z} I_{s}(t, z)=\sigma_{s}^{e} \cdot N_{2}(t, z) \cdot I_{s}(t, z)-\sigma_{s}^{a} \cdot N_{1}(t, z) \cdot I_{s}(t, z) .
\end{gathered}
$$

By eliminating of the populations $N_{1}(t, z)$ and $N_{2}(t, z)$, it results the equivalent system of nonlinear coupled equations:

$$
\begin{gathered}
\frac{\mathrm{d} I_{p}}{\mathrm{~d} z}=-\sigma_{p}^{a} \cdot I_{p} N_{0} \cdot \frac{\frac{\sigma_{p}^{a} \cdot I_{p}}{h v_{p}}+\frac{\sigma_{s}^{a} \cdot I_{s}}{h v_{s}}}{\frac{\sigma_{p}^{a} \cdot I_{p}}{h v_{p}}+\frac{\sigma_{s}^{a} \cdot I_{s}}{h v_{s}}+\frac{1}{\tau}+\frac{\sigma_{s}^{e} \cdot I_{s}}{h v_{s}}}, \\
\frac{\mathrm{d} I_{s}}{\mathrm{~d} z}=\sigma_{s}^{a} \cdot I_{s} N_{0} \cdot\left[\frac{\sigma_{s}^{e}+\sigma_{s}^{a}}{\sigma_{s}^{a}} \cdot \frac{\frac{\sigma_{p}^{a} \cdot I_{p}}{h v_{p}}+\frac{\sigma_{s}^{a} \cdot I_{s}}{h v_{s}}}{\frac{\sigma_{p}^{a} \cdot I_{p}}{h v_{p}}+\frac{\sigma_{s}^{a} \cdot I_{s}}{h v_{s}}+\frac{1}{\tau}+\frac{\sigma_{s}^{e} \cdot I_{s}}{h v_{s}}}-1\right] .
\end{gathered}
$$

In the upper equations, there are involved the parameters: $h=6,626 \cdot 10^{-34} \mathrm{Js}$ - the Planck constant; $c=2,99 \cdot 10^{8} \mathrm{~m} / \mathrm{s}$ - the light velocity in vacuum; $\tau=10^{-2} \mathrm{~s}$ - the relaxation time for spontaneous emission; $\sigma_{p}^{a}=2 \cdot 10^{-16} \mathrm{~m}^{2}$-the absorption cross-section for pumping; $\sigma_{s}^{a}=5 \cdot 10^{-16} \mathrm{~m}^{2}$-the absorption cross-section for signal; $\sigma_{s}^{e}=7 \cdot 10^{-15} \mathrm{~m}^{2}$-the stimulated emission cross-section for signal; $\lambda_{p}=980 \cdot 10^{-9} \mathrm{~m}$ - the pumping radiation wavelength; $\lambda_{s}=1550 \cdot 10^{-9} \mathrm{~m}$ - the signal radiation wavelength; $L$ - the amplifier length; $\Delta z=10^{-3} \mathrm{~m}$ the quantization step in the long of the amplifier. We consider also the parameters: 


$$
\alpha=\left(\frac{h c}{\lambda_{p}}\right)^{-1} ; \beta=\left(\frac{h c}{\lambda_{s}}\right)^{-1} ;\left(\alpha=4,947 \cdot 10^{18} ; \beta=7,824 \cdot 10^{18}\right) .
$$

\subsection{Numerical simulation}

Numerical modeling of the upper rate equations was realized using the MATHLAB programming medium.

The base element of the program was the function ode 45, which realize the integration of the right side expressions of the nonlinear coupled equations using Runge - Kutta type methods, for calculation time reducing.

The program was applied for many values of the amplifier length for each of them resulting different sets of results, for the photon fluxes, both for the signal and pumping as well as for the gain coefficients and signal to noise ratio.

From the obtained results by numerical integration of the transport equations, it results that the intensity of the output signal rise with the amplifier length but the pumping diminish in the some time. The calculated gain coefficients of the amplifier have a similar variation as was expected. We observe also the rising of the signal to noise ratio, resulting an improving of the amplifier performances (Sterian, 2006).

The obtained value of the gain coefficient for the signal, of the $40 \mathrm{~dB}$ is similar to published values (Agrawal, 1995) So that, the results can be very useful for designers, for example, to calculate the optimum length of the amplifier for maximum efficiency.

\section{Laser system in erbium doped active media}

\subsection{The interaction phenomena and parameters}

We analyze the laser systems with $\mathrm{Er}^{3+}$ doped active media by particularizing the models and the method of computer simulation for the case of the $\mathrm{Er}^{3+}$ continuous wave laser which operate on the $3 \mu \mathrm{m}$ wavelength. This laser system is interesting both from theoretical and practical point of view because the radiation with $3 \mu \mathrm{m}$ wavelength is well absorbed in water.

For this type of laser system don't yet completely are known the interaction mechanisms, in spite of many published works.

Quantitative evaluations by numerical simulations are performed, refering to the representative experimental laser with $\mathrm{Er}^{3+}: \mathrm{LiYF}_{4}$, but we analyse also the codoping possibilities of the another host materials: $\mathrm{Y}_{3} \mathrm{Al}_{5} \mathrm{O}_{12}(\mathrm{YAG}), \mathrm{YAIO}_{3}, \mathrm{Y}_{3} \mathrm{Sc}_{2} \mathrm{Al}_{2} \mathrm{O}_{12}$ (YSGG) and $\mathrm{BaY}_{2} \mathrm{~F}_{8}$.

The energy level diagram for the $\mathrm{Er}^{3+}: \mathrm{LiYF}_{4}$ system and the characteristics processes which interest us in that medium are presented in figure 2.

The energy levels of the $\mathrm{Er}^{3+}$ ion include: the ground state in a spectroscopic notation ${ }^{4} I_{15 / 2}$, the first six excited levels ${ }^{4} I_{13 / 2},{ }^{4} I_{11 / 2},{ }^{4} I_{9 / 2},{ }^{4} F_{9 / 2}$, the thermally coupled levels ${ }^{4} S_{9 / 2}+{ }^{2} H_{11 / 2}$ and the level ${ }^{4} F_{7 / 2}$.

The possible mechanisms for operation in continuous wave on $3 \mu \mathrm{m}$ of this type of amplifying media are (Pollnau et al., 1996):

a. the depletion of the lower laser level by absorption in excited state (ESA) ${ }^{4} I_{13 / 2} \rightarrow{ }^{2} H_{11 / 2}$ for pumping wavelength of $795 \mathrm{~nm}$; 


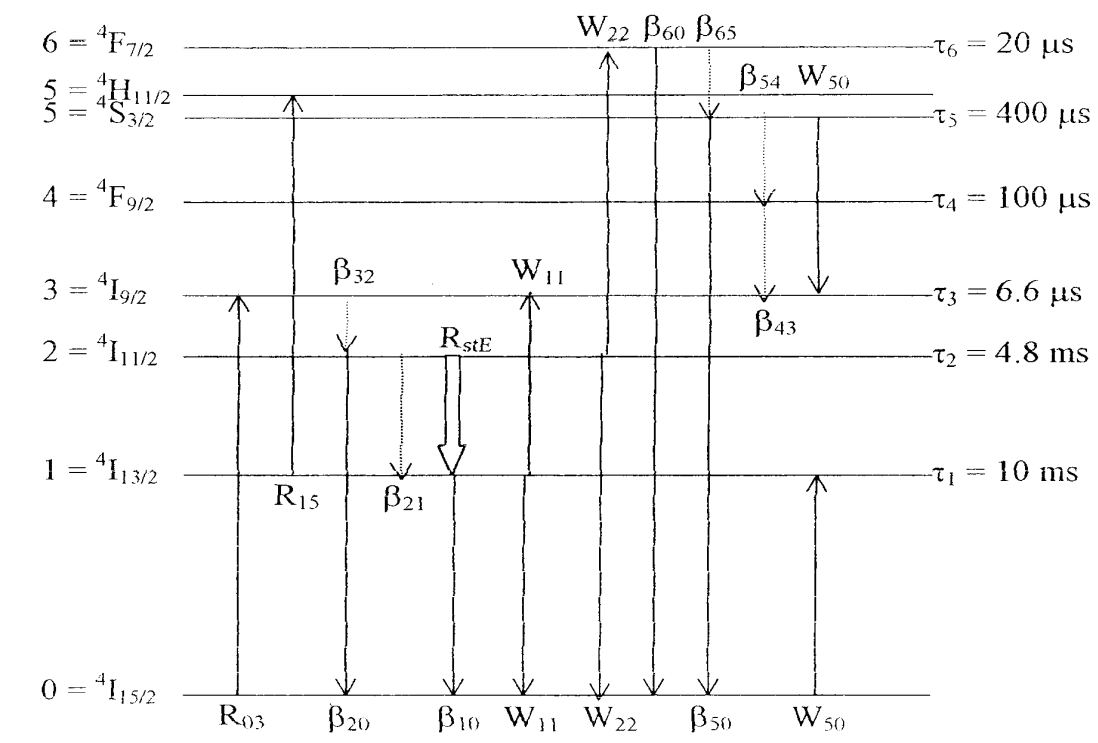

Fig. 2. The energy diagram of the $\mathrm{Er}^{3+}$ ion and the characteristic transitions

b. the distribution of levels excitation ${ }^{4} S_{3 / 2}$ and ${ }^{2} H_{11 / 2}$ between laser levels due to cross relaxation processes $\left({ }^{4} S_{9 / 2}+{ }^{2} H_{11 / 2},{ }^{4} I_{15 / 2}\right) \rightarrow\left({ }^{4} I_{9 / 2},{ }^{4} I_{19 / 2}\right)$ and multiphoton relaxation ${ }^{4} I_{9 / 2} \rightarrow{ }^{4} I_{11 / 2}$;

c. the depletion of the lower laser level and enrichment of the upper laser level due to upconversion processes $\left({ }^{4} H_{13 / 2},{ }^{4} I_{13 / 2}\right) \rightarrow\left({ }^{4} I_{15 / 2},{ }^{4} I_{9 / 2}\right)$ and multiphoton relaxation ${ }^{4} I_{9 / 2} \rightarrow{ }^{4} I_{11 / 2}$

d. the relatively high lifetime for the upper laser level in combination with low branching ratio of the upper laser level to lower laser level.

These mechanisms, separately considered can't explain satisfactory the complex behavior of the erbium doped system, as has been shown (Pollnau et al., 1996; Maciuc et al., 2001).

That is way it is necessary to put into evidence the most important parameters of the system and to clarify the influence of these non-independent parameters on the amplification conditions as well as the determining the optional conditions of operation.

The levels ${ }^{4} \mathrm{H}_{11 / 2}$ and ${ }^{4} S_{3 / 2}$ being thermally coupled, will be treated as combined a level, having a Boltzmann type distribution of the populations.

For numerical simulation the parameters of the $\mathrm{Er}^{3+}: \mathrm{LiYF}_{4}$ were considered because that medium presents a high efficiency for $3 \mu \mathrm{m}$ continuous wave operation, if the pumping wavelength is $\lambda=970 \mathrm{~nm}$ on the upper laser level ${ }^{4} I_{9 / 2}$, or on the level ${ }^{4} I_{11 / 2}$ in the case of the pumping wavelength $\lambda=970 \mathrm{~nm}$.

The Active Medium Parameters. Corresponding to the energy levels diagram presented in figure 2, the lifetimes of the implied levels, for low excitations and dopant concentrations have the values: $\tau_{1}=10 \mathrm{~ms} ; \tau_{2}=4,8 \mathrm{~ms} ; \tau_{3}=6,6 \mu \mathrm{s} ; \tau_{4}=100 \mu \mathrm{s} ; \tau_{5}=400 \mu \mathrm{s}$ and $\tau_{6}=20 \mu \mathrm{s}$.

Just the variations of these intrinsic lifetimes due to ion-ion interactions or ESA will be considered in the rate equations. 
The radiative transitions on the levels ${ }^{4} S_{3 / 2}$ and ${ }^{2} H_{11 / 2}$ are calculated taking into account the Boltzmann contributions of these levels for 300K: 0,935 respectively 0,065 for each transition.

The nonradiative transitions are described through the transition rates $A_{i, N R}$ of the level $i$, calculated with formula:

$$
A_{i, N R}=\tau_{i}^{-1}-\sum_{j=0}^{i=-1} A_{i j},
$$

where $A_{i j}$ are the radiative transition rates from level $i$ to level $j$. In the same time, the branching rations $\beta_{i j}$ of the level i through the another lower levels are given by:

$$
\beta_{i j}=\left(A_{i j}+A_{i, N R}\right) \tau_{i}^{-1}, \text { for } i-j=1
$$

respectively:

$$
\beta_{i j}=\frac{A_{i j}}{\tau_{i}^{-1}}, \text { for } i-j>1 .
$$

The values of the branching ratios have been calculated (Desurvire, 1995; Pollnau et al., 1996).

The considered ion-ion interaction processes are:

$$
\begin{aligned}
& \left({ }^{4} I_{13 / 2},{ }^{4} I_{13 / 2}\right) \leftrightarrow\left({ }^{4} I_{15 / 2},{ }^{4} I_{9 / 2}\right) \\
& \left({ }^{4} I_{11 / 2},{ }^{4} I_{11 / 2}\right) \leftrightarrow\left({ }^{4} I_{15 / 2},\right. \\
& \left.4{ }^{4} I_{7 / 2}\right) \\
& \left({ }^{4} S_{3 / 2},{ }^{2} H_{11 / 2},{ }^{4} I_{15 / 2}\right) \leftrightarrow\left({ }^{4} I_{9 / 2},{ }^{4} I_{13 / 2}\right),
\end{aligned}
$$

being characterized by the next values of the transition rates:

$$
\begin{gathered}
W_{11}=W_{11}^{-1}=3 \cdot 10^{-23} \mathrm{~m}^{3} \mathrm{~s}^{-1} ; W_{22}=W_{22}^{-1}=1,8 \cdot 10^{-23} \mathrm{~m}^{3} \mathrm{~s}^{-1} \\
W_{50}=W_{50}^{-1}=2 \cdot 10^{-23} \mathrm{~m}^{3} \mathrm{~s}^{-1}
\end{gathered}
$$

where the $W_{50}$ parameter take into account the indiscernible character of the corresponding relaxation processes.

The Resonator Parameters. The resonator parameters used in the realized computer experiments are consistent with operational laser systems, as: the crystal length: $l=2 \mathrm{~mm}$; the dopant concentration: $N_{0}=2 \cdot 10^{21} \mathrm{~cm}^{-3}$; the pumping wavelength: $\lambda_{p}=795 \mathrm{~nm}$; we consider for ground state absorption (GSA) ${ }^{4} I_{15 / 2} \rightarrow{ }^{4} I_{9 / 2}$ the cross section $\sigma_{03}=5 \cdot 10^{-21} \mathrm{~cm}^{2}$ and for excited state absorption (ESA), ${ }^{4} I_{13 / 2} \rightarrow{ }^{4} S_{3 / 2}+{ }^{2} H_{11 / 2}$, the cross section $\sigma_{15}=1 \cdot 10^{-20} \mathrm{~cm}^{2}$.

(The ESA contribution of the level ${ }^{4} I_{11 / 2}$ was neglected for that wavelength.)

Another considered parameter values are presented in literature, being currently used by researchers. 
In the literature (Pollnau et al., 1996; Maciuc et al., 2001). we found also the values of the energy levels populations reported to the dopant concentration and the relative transition rates, for different wavelength used for pumping: $\lambda=795 \mathrm{~nm}$ and $\lambda=970 \mathrm{~nm}$.

\subsection{Computational model}

The presented model, include eight differential equations which describes the population densities of each $\mathrm{Er}^{3+}$ ion energy levels presented in figure 2 and the photon laser densities inside the laser cavity.

We take $N_{i}$ for $i=1,2, \ldots, 6$ to be the population density of the $i$ level and $N_{0}$ the population density of the ground state, the photonic density being $\phi$.

That model consisting of eight equation system is suitable for crystal laser description (Pollnau et al., 1994). For the fiber laser, the model must be completed with a new field equation to describe the laser emission on $\lambda=1,7 \mu \mathrm{m}$ between the fifth and the third excited levels.

The rate equations corresponding to energy diagram with seventh levels, for $\mathrm{Er}^{3+}$ systems are presented below:

$$
\begin{gathered}
\frac{\mathrm{d} N_{6}}{\mathrm{~d} t}=\sum_{i=0}^{5} R_{i 6} N_{i}-\tau_{6}^{-1} N_{6}+W_{22}\left(N_{2}^{2}-N_{0} N_{6}\right) \\
\frac{\mathrm{d} N_{5}}{\mathrm{~d} t}=\sum_{i=0}^{4} R_{i 5} N_{i}-R_{56} N_{5}-\tau_{5}^{-1} N_{5}+\beta_{56} \tau_{6}^{-1} N_{6}-W_{50}\left(N_{5} N_{0}-N_{3} N_{1}\right)-R_{S E}^{5-3} \\
\frac{\mathrm{d} N_{4}}{\mathrm{~d} t}=\sum_{i=0}^{3} R_{i 4} N_{i}-\sum_{j=5}^{6} R_{4 j} N_{4}-\tau_{4}^{-1} N_{4}+\sum_{i=4}^{6} \beta_{i 4} \tau_{i}^{-1} N_{i} \\
\frac{\mathrm{d} N_{3}}{\mathrm{~d} t}=\sum_{i=0}^{3} R_{i 3} N_{i}-\sum_{j=4}^{6} R_{3 j} N_{3}-\tau_{3}^{-1} N_{3}+ \\
+\sum_{i=4}^{6} \beta_{i 3} \tau_{i}^{-1} N_{i}+W_{50}\left(N_{5} N_{0}-N_{3} N_{1}\right)+W_{11}\left(N_{1}^{2}-N_{0} N_{3}\right)+R_{S E}^{5 \rightarrow 3} \\
\frac{\mathrm{d} N_{2}}{\mathrm{~d} t}=\sum_{i=0}^{1} R_{i 2} N_{i}-\sum_{j=3}^{6} R_{2 j} N_{2}-\tau_{2}^{-1} N_{2}+ \\
+\sum_{i=3}^{6} \beta_{i 2} \tau_{i}^{-1} N_{i}-2 W_{22}\left(N_{2}^{2}-N_{0} N_{6}\right)-R_{S E} ; \\
\frac{\mathrm{d} N_{1}}{\mathrm{~d} t}=R_{01} N_{0}-\sum_{j=2}^{6} R_{1 j} N_{1}-\tau_{1}^{-1} N_{1}+N_{i}+W_{50}\left(N_{5} N_{0}-N_{3} N_{1}\right)-2 W_{11}\left(N_{1}^{2}-N_{0} N_{3}\right)+R_{S E} ;
\end{gathered}
$$




$$
\begin{gathered}
\frac{\mathrm{d} N_{0}}{\mathrm{~d} t}=-\sum_{j=0}^{6} R_{0 j} N_{0}+\sum_{i=1}^{6} \beta_{i 0} \tau_{i}^{-1} N_{i}-W_{50}\left(N_{5} N_{0}-N_{3} N_{1}\right)+ \\
+W_{11}\left(N_{1}^{2}-N_{0} N_{3}\right)+W_{22}\left(N_{2}^{2}-N_{0} N_{6}\right) . \\
\frac{\mathrm{d} \phi}{\mathrm{d} t}=\frac{l}{l_{\text {opt }}}\left(\frac{P_{l}}{P} \gamma_{21} \beta_{21} \tau_{2}^{-1} N_{2}+R_{S E}\right)-\left\{-\ln \left[(1-T)\left(1-L_{r}\right)\right]+2 \kappa l\right\} \frac{c \phi}{2 l_{o p t}} ; \\
\frac{\mathrm{d} \phi^{5 \rightarrow 3}}{\mathrm{~d} t}=\frac{l}{l_{\text {opt }}}\left(\frac{P_{l}}{P} \gamma_{53} \beta_{53} \tau_{5}^{-1} N_{5}+R_{S E}^{5 \rightarrow 3}\right)-\left\{-\ln \left[(1-T)\left(1-L_{r}\right)\right]+2 \kappa l\right\} \frac{c \phi^{5 \rightarrow 3}}{2 l_{o p t}} .
\end{gathered}
$$

A similar models are given in the references ( Pollnau et al., 1996; Maciuc et al., 2001,a \& b). In the field equations (27) and (28), the parameters $L, T, L_{r}, \kappa, l_{\text {opt }} \cdot P_{l} / P$ are considered the same for the two type of laser studied. In the equations system $(20) \div(28)$ the parameters are: $R$ is the pumping rate from lower levels to the higher ones; $\tau$ is the life-times for each corresponding level; $W$ is associated with the transition rates of the ion-ion up-conversion and the corresponding inverse processes; - $\beta_{i j}$ are the branching ratios of the level $i$ through the other possible levels $j ; R_{S E}$ is the stimulated emission rate; $l$ and $l_{\text {opt }}$ are the crystal length and the resonator length; $\gamma_{21}$ is an additional factor for the spontaneous radiative transition fraction between the levels: ${ }^{4} I_{11 / 2}$ and ${ }^{4} I_{13 / 2} ; P_{l} / P$ is the of spontaneous emission power emitted in laser mode; $T, L_{r}, \kappa, c$ are the transmission of the output coupling mirror, the scattering losses and the diffraction - reabsorption losses respectively, $c$ being the light speed in vacuum.

The pumping rates depend on the corresponding cross-section and of the other parameters (Maciuc et al., 2001).

The parameters for the lasing in an Er:LiYF 4 crystal system are considered the same and for the fiber laser.

\subsection{Crystal laser simulation}

Laser Efficiency for Different Pumping Wavelength. In the simulation were used for pumping the radiations having $\lambda=795,970$ and $1570 \mathrm{~nm}$, which are in resonance with the energy levels in diagram of $\mathrm{Er}^{3+}$ ion presented in figure 2.

The pumping radiation for $\lambda=795 \mathrm{~nm}$ connect the ground state level ${ }^{4} I_{15}$ with the third excited level ${ }^{4} I_{9 / 2}$ and also the second level with the fifth one $\left({ }^{4} I_{13 / 2},{ }^{4} S_{3 / 2}+{ }^{2} I_{11 / 2}\right)$, processes.

In the case of pumping radiation having $\lambda=970 \mathrm{~nm}$ the ground state absorption (GSA) corresponds to transition ${ }^{4} I_{15} \rightarrow{ }^{4} I_{11 / 2}$ and excited state absorption (ESA) to transition ${ }^{4} I_{11 / 2} \rightarrow{ }^{4} F_{7 / 2}$. Similarly the pumping for $\lambda=1530 \mathrm{~nm}$ determine a single transition GSA that is ${ }^{4} I_{15 / 2} \rightarrow{ }^{4} I_{13 / 2}$.

The dependence of the output power versus input power for different pumping wavelength (795 nm, $970 \mathrm{~nm}$ and $1530 \mathrm{~nm}$ ) were plotted resulting the functioning thresholds and the slope efficiencies for each situation.

For the crystal laser $\mathrm{Er}^{3+}$ doped, the optimum efficiency results for the direct pumping on the upper laser level. 
The output power variation with the level lifetimes. The output power variation on the lifetimes for the upper levels having $\tau_{4}, \tau_{5}, \tau_{6}$ was studied for an input pump power $P_{p}=5 \mathrm{~W}$ and $\lambda_{p}=795 \mathrm{~nm}$.

We found that radiative and nonradiative transitions from the fifth and the sixth levels, improve the population difference for the laser line and determine the raising of the output power of them, the variation of the fourth level lifetime, being without influence for the output power.

The influence of the $\mathrm{Er}^{3+}$ ion doped host material on the output power. A three dimensional study was done to investigate the influence in the laser output power due to parameters variations for the host material, using $\lambda_{p}=795 \mathrm{~nm}$.

The relative spontaneous transition rates were considered the same for all simulations.

To determine the host material change influence on the laser output power the next variation scale of the lifetimes have been considered:

$$
\begin{gathered}
\tau_{1}=(1 \div 15) \mathrm{ms}, \tau_{2}=(0,4 \div 9,6) \mathrm{ms}, \tau_{3}=(0,22 \div 22) \mu \mathrm{s}, \tau_{4}=(3 \div 300) \mu \mathrm{s}, \tau_{5}=(12 \div 1200) \mu \mathrm{s} \\
\text { and } \tau_{6}=(0,6 \div 60) \mu \mathrm{s} .
\end{gathered}
$$

Similarly, the variations of the transition rates corresponding to up-conversion processes for different host materials are considered to span the intervals given bellow:

$$
\begin{gathered}
W_{11}=(0,1 \div 300) \cdot 10^{-21} \mathrm{~cm}^{3} \mathrm{~ms}^{-1}, W_{22}=(1,8 \div 180) \cdot 10^{-21} \mathrm{~cm}^{3} \mathrm{~ms}^{-1} \\
W_{50}=(0,02 \div 200) \cdot 10^{-21} \mathrm{~cm}^{3} \mathrm{~ms}^{-1}
\end{gathered}
$$

For the other parameters used in the numerical simulation the published data was the main source of reference.
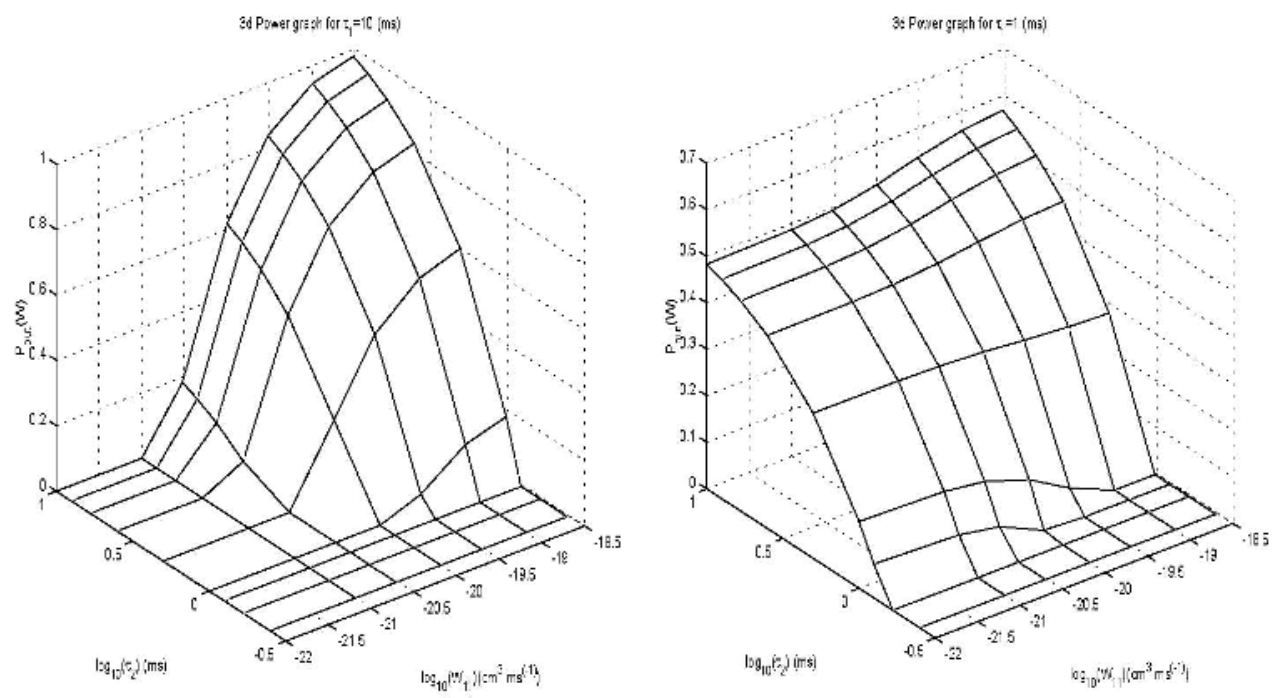

Fig. 3. Output laser power dependence on parameters $\tau_{2}$ and $W_{11}$ for two values of $\tau_{1}$ 
The graphs in figure 3 give the three-dimensional (3D) output power dependence versus lifetime of the higher level $\tau_{2}$ and the "up-conversion" parameter $W_{11}$ associated to studied transition for two values of the $\tau_{1}$ parameter: $\tau_{1}=.10 \mathrm{~ms}$ and $\tau_{1}=1 \mathrm{~ms}$. In figure 4 we show the dependence of the output power on the life time of the second excited level, the "up-conversion" parameter $W_{11}$ and "up-conversion parameter $W_{22}$ for two values of this parameter: $W_{22}=1,8 \cdot 10^{-24} \mathrm{~m}^{3} \mathrm{~s}^{-1}$ and $W_{22}=1,8 \cdot 10^{-22} \mathrm{~m}^{3} \mathrm{~s}^{-1}$.
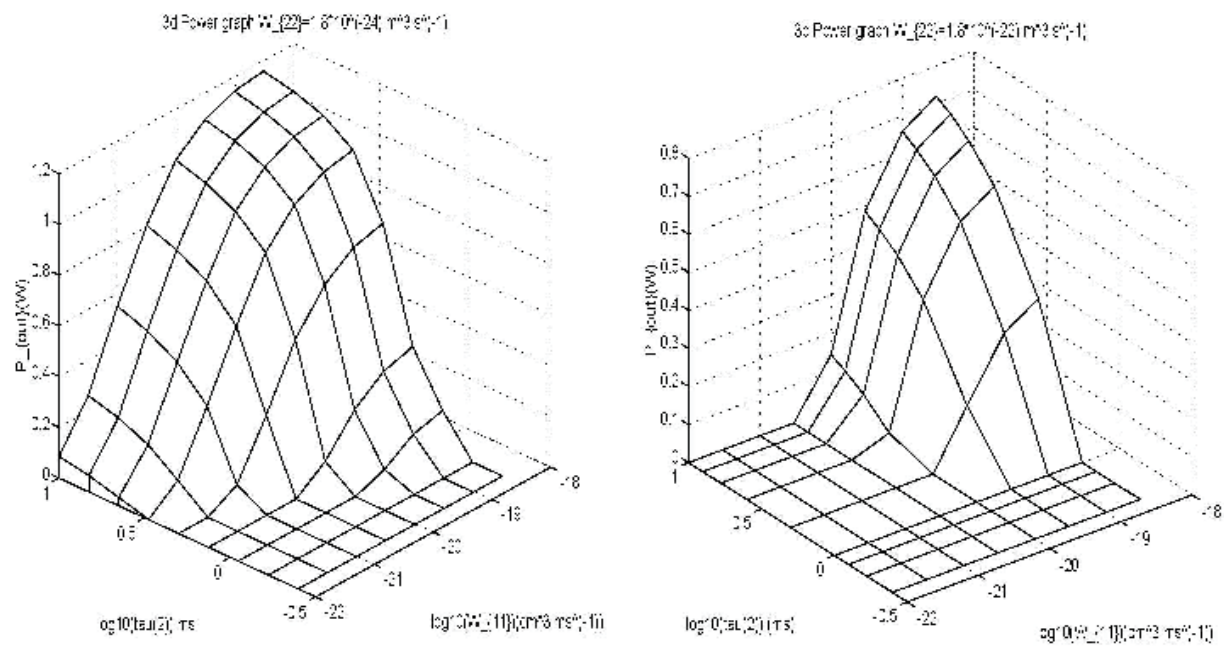

Fig. 4. Output laser power dependence on parameters $\tau_{2}$ and $W_{11}$ for two values of $W_{22}$

The graphs in figure 5 give a three-dimensional representation of the function $P_{\text {laser }}\left(\tau_{3}, W_{11}, \tau_{1}\right)$ for two values of the $\tau_{1}$ parameter: $\tau_{1}=.10 \mathrm{~ms}$ and $\tau_{1}=1 \mathrm{~ms}$.

The three-dimensional study of the parameters variation to increase laser output power showed the role of host material for high laser efficiency, checking that the decisive parameter is the time of life associated with the upper laser level. Selection of the materials having parameters in the areas of variation adopted in the analysis recommend as efficient solutions the fluorides: LiYF4 and BaY2F.

Stable, non-chaotic behavior of the laser systems. A time dependence of the photon density in the cavity of the output power and of the implied level populations in the laser process was analyzed by the input parameters variations that is pumping power and the interaction cross-sections. For the pumping power differently step functions was considered. The analysis represents a satisfactory temporally description of the crystal laser to verify the used computational model.

Our simulation for the time dependence confirm the stability of the continuous wave regime of operation of the crystal laser, after an initial transitory regime of the milliseconds order, which is gradually droped, from the moment we switch on the pump.

This stable non-chaotic behavior is similar for different host materials, the used method not being time prohibitive for such studies.

To understand better the obtained results, we indicate below some of the graphs plotted in that simulation: $3 \mathrm{~d}$ analysis $P\left(W_{11}, \tau_{1}, \tau_{2}\right)$ with $\tau_{1}=10 \mathrm{~ms} ; 3 \mathrm{~d}$ analysis $P\left(\sigma_{15}, \tau_{2}, W_{11}\right)$ with $\sigma_{15}=10^{-19} \mathrm{~cm}^{2} ; 3 \mathrm{~d}$ analysis $P\left(W_{50}, \tau_{2}, W_{22}\right)$ with $W_{22}=1,8 \cdot 10^{-24} \mathrm{~m}^{3} \mathrm{~s}^{-1} ; 3 \mathrm{~d}$ analysis $P\left(W_{50}, \tau_{1}, \tau_{3}\right)$ with $\tau_{1}=10 \mathrm{~ms}$, etc. 

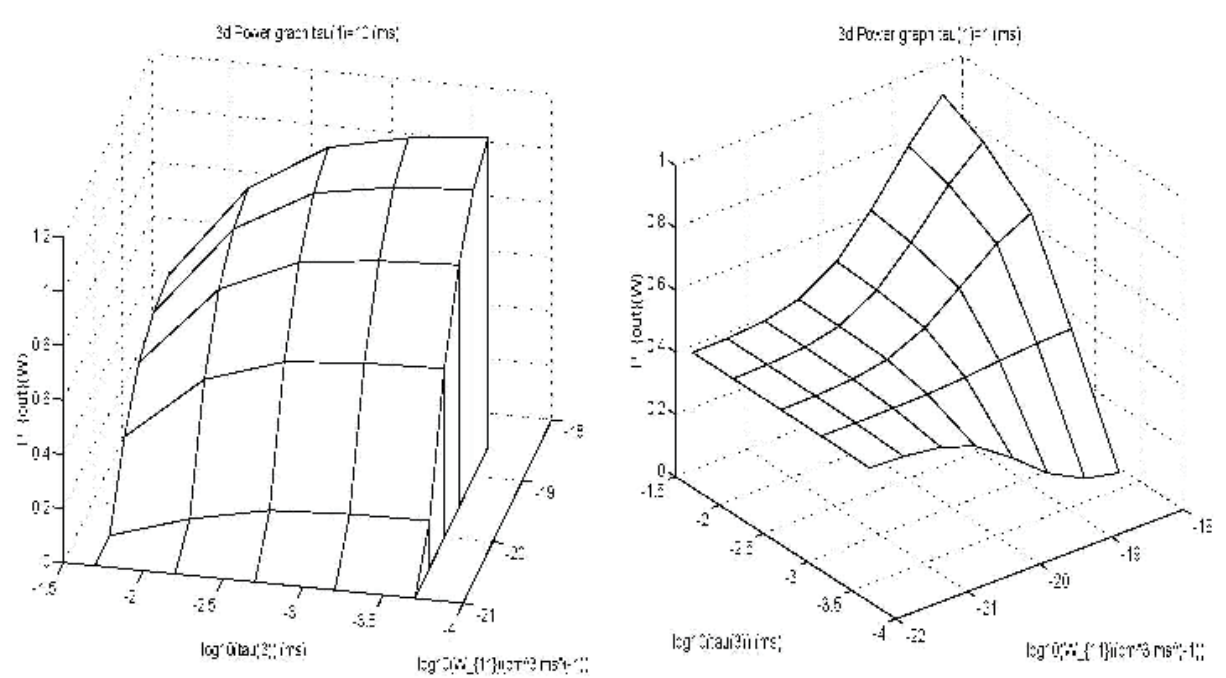

Fig. 5. Output laser power dependence on parameters $\tau_{3}$ and $W_{11}$ for two values of $\tau_{1}$

In conclusion, the 3D study of the parameters variations to rise the output laser power put intro evidence the important role of the host materials, the decisively parameter being the lifetime associated with the upper laser level.

By selection, other the parameters variations limits, the most efficiently media are the fluorides: $\mathrm{LiYF}_{4}$ and $\mathrm{LiY}_{2} \mathrm{~F}_{8}$.

In spite of the fact we have analyzed the problems by an original method, the results are consistent with the published data.

A special mention must be made concerning the used "step-size" Runge - Kutta method which is rapidly and don't alterate the results obtained by classical Runge - Kutta method.

In case of $3 \mathrm{~d}$ analysis we used a 7 order precision and a 6 order stopping criteria.

\subsection{Fiber laser simulation}

In the fiber laser functioning, were studied almost the same problems as in the crystal laser case, that are:

a. The output power thresholds and efficiencies for different values of the "colaser" process and in the absence of this effect.

b. The relevance and the implications of the "colaser" process, which is specific to fiber laser

c. The dependence of the output power on host material $\mathrm{Er}^{3+}$ doped, by variation of the characteristic parameters.

d. The description of the time depended phenomena for the $\mathrm{Er}^{3+}$ doped fiber laser, inclusively the population dynamics.

The principal differences between the crystal laser and fiber laser were taken into consideration, the most important being:

- the existence of an extra field equation (Maciuc et al., 2001), which describes the colasing process in the fiber laser;

- the absence of the "up-conversion" processes due to the low concentration of the $\mathrm{Er}^{3+}$ dopant. 
The role played by the up-conversion in crystal is taken in fiber laser by pumping from the first and second excited level.

The analyzed physical system was the optical fiber with ZBLAN composition, having the next characteristic parameters:

- the dopant concentration: $N_{d}: 1,8 \cdot 10^{19} \mathrm{~cm}^{-3}$; the amplifier length, $l: 480 \mathrm{~cm}$; the laser mod radius, $r_{\text {mode }}: 3,25 \mu \mathrm{m}$; the pumping wavelength, $\lambda_{p}: 791 \mathrm{~nm}$; the ground state absorption cross-section, $\sigma_{03}: 4,7 \cdot 10^{-22} \mathrm{~cm}^{2}$; the excited state absorption cross-section from the level ${ }^{4} I_{13 / 2}, \sigma_{15}: 10^{-21} \mathrm{~cm}^{2}$; the excited state absorption cross-section from the level ${ }^{4} I_{11 / 2}, \quad \sigma_{27}: 2 \cdot 10^{-22} \mathrm{~cm}^{2}$; the laser wavelength, $\lambda_{L}: 2,71 \mu \mathrm{m}$; the "colaser" wavelength, $\lambda_{c l}: 1,7 \mu \mathrm{m}$; the emission cross-section, $\sigma_{21}: 5,7 \cdot 10^{-21} \mathrm{~cm}^{2}$; the "colaser" cross section, $\sigma_{53}: 0,5$ or $0,1 \cdot 10^{-20} \mathrm{~cm}^{2}$; the Boltzmann, $b_{14}$ and $b_{22}: 0,113$ respectively 0,2; the mirror transmission $T: 68 \%$; the optical resonator length, $l_{\text {opt }}: 720 \mathrm{~cm}$.

The "colaser" process was studied for three different values of the "colaser" cross section: $\sigma_{53}=0 \mathrm{~cm}^{2} ; \sigma_{53}=0,5 \cdot 10^{-20} \mathrm{~cm}^{2}$ and $\sigma_{53}=0,1 \cdot 10^{-20} \mathrm{~cm}^{2}$.

The most important conclusions resulting from the fiber laser analysis are:

- The optimum operating conditions are obtained for $\lambda_{p}=791 \mathrm{~nm}$, so that the pumping is realized directly on the upper laser level with the cross - section $\sigma_{03}$.

- The presence of the "colaser" process, improves the laser efficiency on $3 \mu \mathrm{m}$, by a 2 factor in that cascade laser situation. The three - dimensional (3D) analysis shows the determinant role of the $\tau_{2}$ for laser power similarly to the crystal laser, the parameters $\sigma_{15}$ and $\sigma_{27}$ being strong correlated with the laser process, for the high values of the $\tau_{11}$.
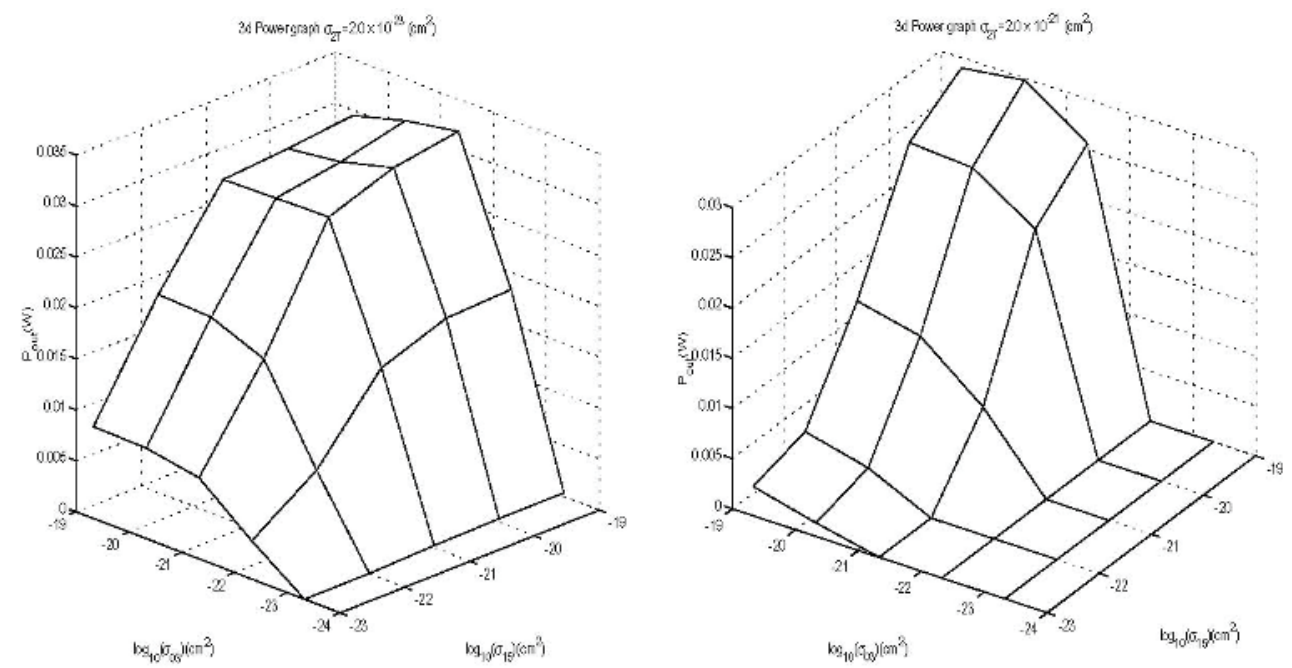

Fig. 6. Dependence of the output laser power both on $\sigma_{15}$ and $\sigma_{03}$ cross sections for two values of $\sigma_{27}$.

The graphs in Figure 6 shows a strong dependence of the laser power both on $\sigma_{15}$ and $\sigma_{03}$ cross sections in terms of the high life time of the lower laser level $\tau_{1}$ and a sufficiently low value of the effective cross section, $\sigma_{27}=2 \cdot 10^{-23} \mathrm{~cm}^{2}$. Increasing the value of the second 
process(ESA) (Fig. 7) on obtain the same behavior of the output power with the difference of a rapid increase from zero to a high value of a laser power, followed by a slow saturation.
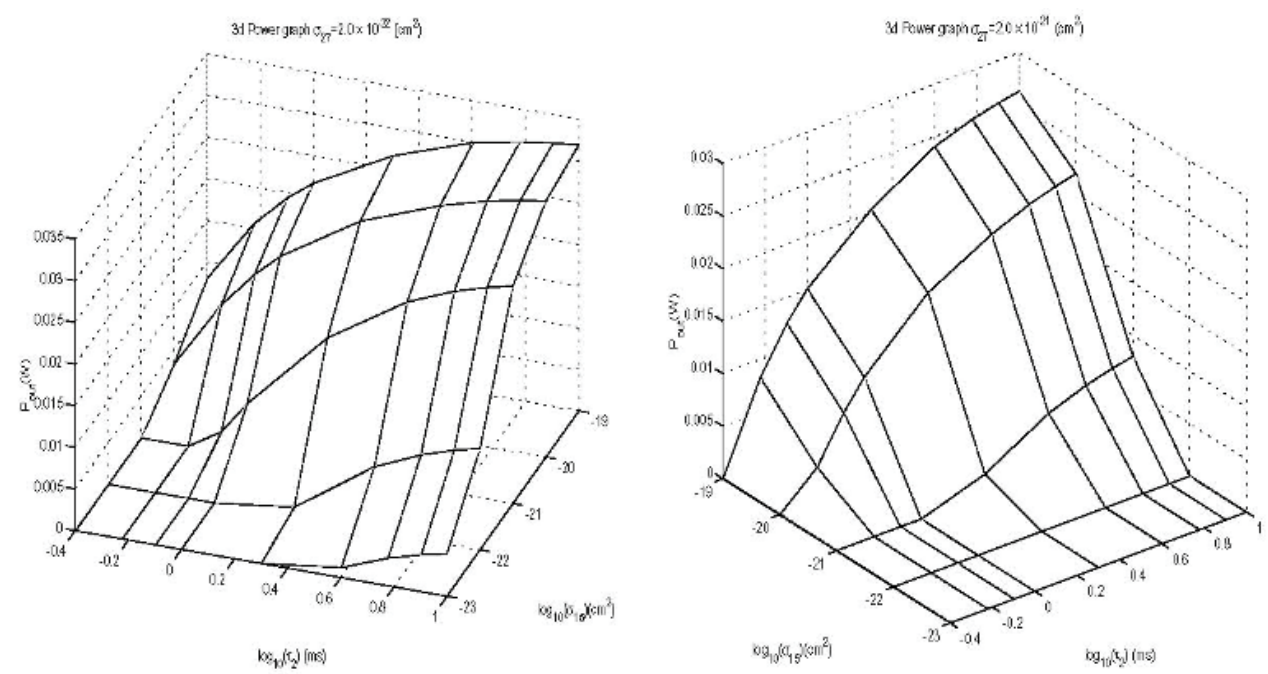

Fig. 7. Dependence of the output laser power both on $\sigma_{15}$ and $\tau_{2}$ for two values of $\sigma_{27}$

The dependence $P\left(\sigma_{15}, \tau_{2}, \sigma_{27}\right)$ represented by the graphs in figure 7 , leads to the conclusions:

- increasing of $\tau_{2}$ the laser power increases;

- the rising of $\sigma_{15}$ is favorable to laser power for $\tau_{1}=10 \mathrm{~ms}$;

- high values of $\sigma_{27}$ causes low laser powers, due to the depopulation of the upper laser level. The study of the "colaser" process was made for three different values of effective "colaser" cross-section: $\sigma_{53}=0 \mathrm{~cm}^{2} ; \sigma_{53}=0,5 \cdot 10^{-20} \mathrm{~cm}^{2}$ and $\sigma_{53}=0,1 \cdot 10^{-20} \mathrm{~cm}^{2}$. (Note that ZBLAN fiber optic amplifiers require more pumping power than EDFA's based on silicon dioxide.)

Another important result is represented by the time dependent analysis of the output power and of the level populations, which shows a stable non - chaotic behavior as in the crystal laser case.

All obtained results by numerical analysis are consistent with the data from the literature (Shalibeik, 2007).

\section{Nonlinear effects in optical fibers systems.}

\subsection{The model}

In recent years much attention has been paid to the study of nonlinear effects in optical fiber lasers (Agrawal, 1995 \& 1997; Desurvire, 1995).

Self-pulsing and chaotic operation (Baker \& Gollub, 1990; Abarbanel, 1996) of the EDFLs has been reported in various experimental conditions (Sanchez et al., 1993; Sanchez et al., 1995) including the case of pumping near the laser threshold. We present firstly a model for the single-mode laser taking into account the presence of the erbium ion pairs that act as a saturable absorber. 
The nonlinear dynamics of an erbium-doped fiber laser is explained based on a simple model of the ion pairs present in heavily doped fibers. The single-mode laser dynamics is reducible to four nonlinear differential equations. Depending on the ion pair concentration, the pumping level and the photon lifetime in the laser cavity, numerical calculations predicts $\mathrm{cw}$, self-pulsing and sinusoidal dynamics. The regions of these dynamics in the space of the laser parameters are determined.

A modeling of the erbium laser operating around $1.55 \mu \mathrm{m}$ has been proposed (Sanchez et al., 1993). This considers the amplifying medium as a mixture of isolated erbium ions and erbium ion pairs. For an isolated ion, the laser transition takes place between the energy levels ${ }^{4} I_{13 / 2}$ and ${ }^{4} I_{15 / 2}$ (Fig. 8). The ion is pumped on some upper energy levels and it fastly relaxes to the level ${ }^{4} I_{13 / 2}$. It is noteworthy that energy level ${ }^{4} I_{9 / 2}$ is positioned above level ${ }^{4} I_{13 / 2}$ at a separation approximately equal to that of the laser transition. Two neighboring ions interact and form an ion pair. The strength of this interaction is small (due to the screening effect of the $4 d^{10}$ electrons on the $4 f$ electrons) so that the energy levels are practically preserved and the pair energy is the sum of the two ions energy. Because of the quasiresonance of levels ${ }^{4} I_{9 / 2}$ and ${ }^{4} I_{13 / 2}$ with the laser transition, up-conversion in an ion pair has a significant probability. This is followed by a fast transition back to the ${ }^{4} I_{13 / 2}$ level. As a result of these processes, the population inversion decreases by one without the emission of a photon. Thus, the laser effect due to the ion pair is explained based on three ionic levels.

Based on the above picture of the active medium, in the rate equation approximation the laser is described by the population inversion $d$ of the isolated ions, the sum $d_{+}$and the difference $d_{-}$of populations of levels 22 and 11, and the normalized laser intensity $I$, that verify equations (Flohic et al., 1991; Sanchez et al., 1993):

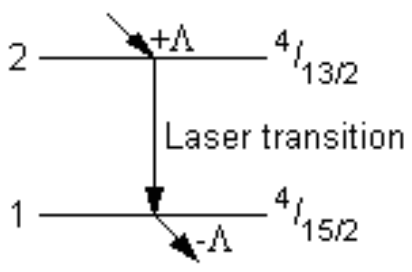

(a)

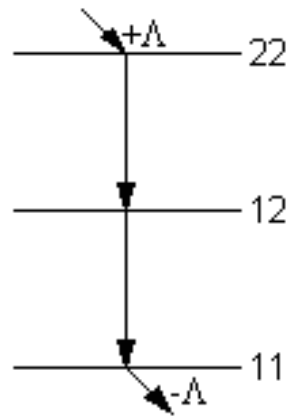

(b)

Fig. 8. Laser energy levels: (a) an isolated erbium ion and (b) an ion pair.

$$
\begin{gathered}
\dot{d}=\Lambda-a_{2}(1+d)-2 d I, \\
\dot{d}_{+}=a_{2}\left(1-d_{+}\right)-\left(a_{22} / 2\right)\left(d_{+}+d_{-}\right)+y\left(2-3 d_{+}\right) I, \\
\dot{d}_{-}=\Lambda-a_{2}\left(1-d_{+}\right)-\left(a_{22} / 2\right)\left(d_{+}+d_{-}\right)-y d_{-} I,
\end{gathered}
$$




$$
\dot{I}=-I+A(1-2 x) d I+A x y d_{-} I .
$$

In the above, the time variable is expressed in units of the photon lifetime in the cavity $\left(\tau_{\mathrm{L}}\right)$. The quantity $x$ is the fraction of ion pairs in the active medium, $\Lambda$ is the pumping parameter, $a_{2}=\tau_{\mathrm{L}} / \tau_{2}$ and $a_{22}=\tau_{\mathrm{L}} / \tau_{22}$, where $\tau_{2}$ and $\tau_{22}$ are the lifetimes of level 2 and 22 , respectively. The other parameters are $y=\sigma_{\mathrm{L}}^{\prime} / \sigma_{\mathrm{L}}$, where $\sigma_{\mathrm{L}}$ and $\sigma_{\mathrm{L}}^{\prime}$ are the absorption cross-section for the laser transition in isolated ions and pairs, respectively, and $A=\sigma_{\mathrm{L}} N_{0} \tau_{\mathrm{L}}$, where $N_{0}$ is the erbium concentration. The normalization of the laser intensity $I$ is performed in the form $I=i_{\mathrm{L}} / \sigma_{\mathrm{L}} \tau_{\mathrm{L}}$.

\subsection{Stability analysis}

Apart from the nonlasing state (zero intensity state), the intensity $i_{\mathrm{L}}$ in a stationary state satisfies the third-order polynomial equation:

$$
i_{\mathrm{L}}^{3}+c_{1} i_{\mathrm{L}}^{2}+c_{2} i_{\mathrm{L}}+c_{3}=0
$$

where:

$$
\begin{gathered}
c_{1}=A x\left(a_{22}-2 a_{2}\right) / 3+\left(a_{2}+2 a_{22}\right) / 3 y+a_{2} / 2-A\left(2 \Lambda-a_{2}\right) / 2, \\
c_{2}=x\left[a_{2} A\left(a_{2} / 6+a_{22} / 6-\Lambda\right)+a_{22} A \Lambda / y-a_{2} A\left(a_{2}+a_{22}\right) / 3 y\right] \\
+a_{2}\left(a_{2}+2 a_{22}\right) / 6 y+a_{2} a_{22} / 3 y^{2}-A\left(a_{2}+2 a_{22}\right)\left(2 \Lambda-a_{2}\right) / 6 y, \\
c_{3}=a_{2} A x\left(a_{2} a_{22}-2 a_{2} \Lambda-a_{22} \Lambda\right) / 6 y+a_{2}^{2} a_{22} / 6 y^{2}-a_{2} a_{22} A(1-2 x)\left(2 \Lambda-a_{2}\right) / 6 y^{2} .
\end{gathered}
$$

The laser threshold is derived from the condition $c_{3}=0$ that implies:

$$
\Lambda_{\mathrm{th}}=\frac{1-A x(2-y) /(A+1)}{1-\left(2-y / 2-a_{2} y / a_{22}\right) x} \Lambda_{\mathrm{th}}^{0}
$$

where:

$$
\Lambda_{\mathrm{th}}^{0}=(1+1 / A) a_{2} / 2
$$

is the pumping parameter at laser threshold in the absence of the ion pairs. Numerical solution of eq. (5) is performed for typical parameters. We take $\tau_{2}=10 \mathrm{~ms}, \tau_{22}=2 \mu \mathrm{s}$, $N_{0}=5 \times 10^{24} \mathrm{~m}^{-3}, \sigma_{\mathrm{L}}=1.6 \times 10^{-16} \mathrm{~m}^{2}$ and $y=0.2$. The dependence of the threshold pumping level on the concentration of the ion pair for two values of photon lifetime in the cavity is presented in Fig. 9. The increase of the laser threshold due to the presence of the ion pairs limits the use of the fibers in such conditions.

Above threshold there exists one steady-state intensity given by eq. (5), the other two solutions being unphysical (negative). The numerical calculation of the intensity in a significant range of the pumping parameter (Fig.10) gives a laser intensity following a straight line dependence. The influence of the ion pairs is again disadvantageous and manifests in reducing the slope of the characteristics. 


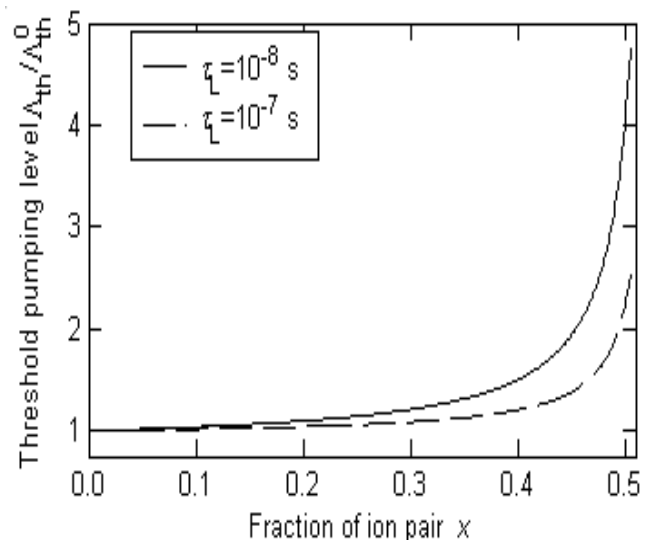

Fig. 9. Threshold pumping parameter vs. ion pair percentage

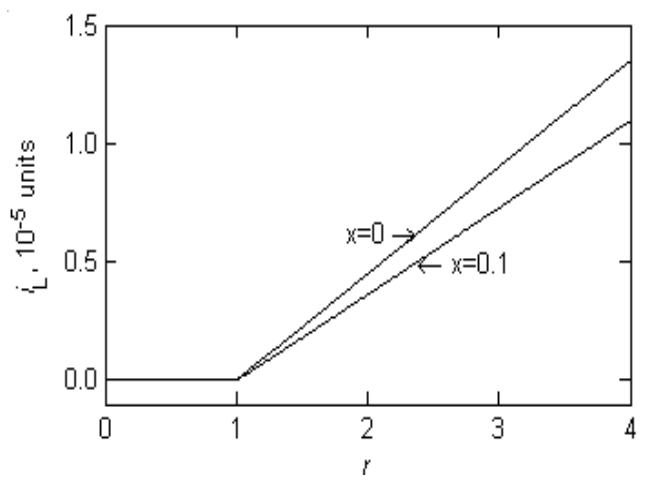

Fig. 10. Laser intensity in the steady state versus the pumping strength
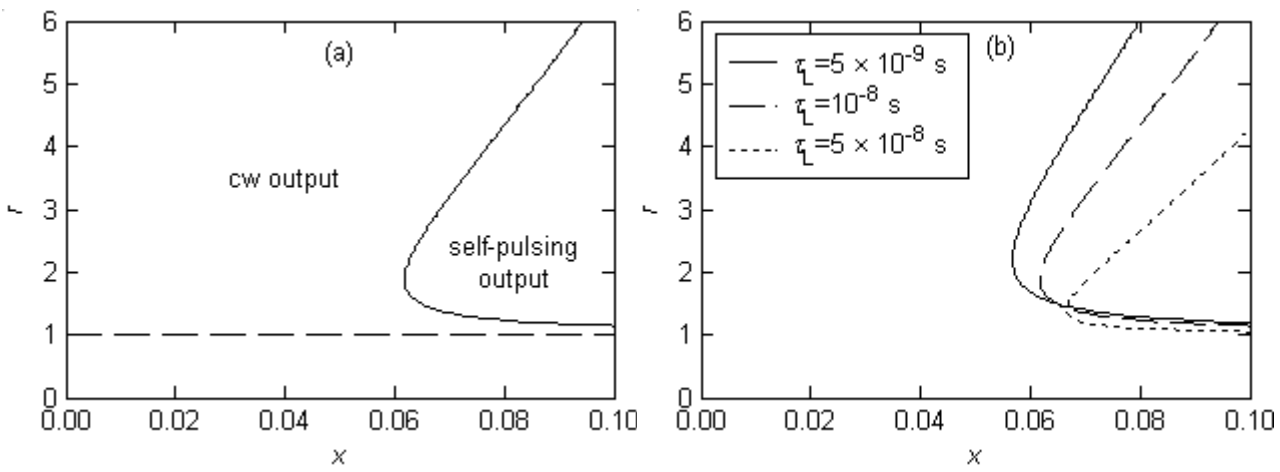

Fig. 11. (a) Calculated stability diagram for EDFL. $\tau_{\mathrm{L}}=10^{-8} \mathrm{~s}$; (b) The influence of the photon lifetime on the margins of the stability domains. 

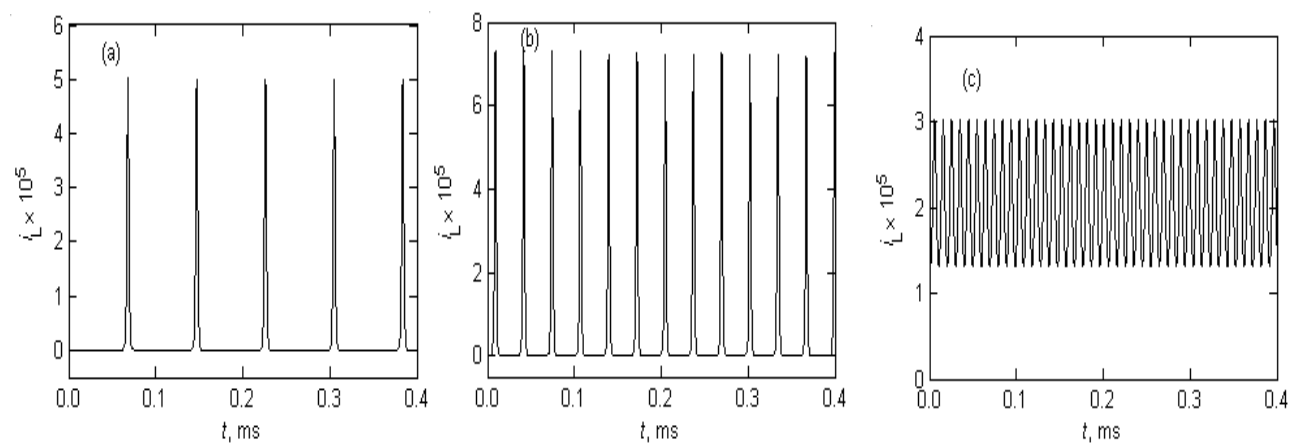

Fig. 12. Long term temporal evolution of laser intensity at the fraction $x=0.1$ of ion pairs and the pumping level (a) $r=1.5$, (b) $r=2.5$, and (c) $r=6.6 . \tau_{\mathrm{L}}=10^{-8} \mathrm{~s}$.

Linear stability investigation of the steady states (Crawford, 1991, Press, 1992) reveals the existence of a critical value of the ion pair percentage under which the steady state $(\mathrm{cw})$ solution is stable whatever the pumping level. At larger concentrations of the ion pairs, the laser is in a steady state or self-pulsing, depending on the value of the pumping parameter (Fig. 11.a). The transition from the cw dynamics to a self-pulsing one takes place when two complex conjugate eigenvalues of the linearized system cross the imaginary axis from left to right, i.e., a Hopf bifurcation occurs.( Crawford, 1991). In such conditions the steady state solution becomes unstable and the long-term system evolution settle down on a stable limit cycle in phase space.

The photon lifetime contribution to the stability diagram is presented in Fig. 11.b. This proves that a cavity with low losses makes it possible to preserve the cw dynamics at larger doping levels.

Fig.12 clarifies the quantitative changes of the laser intensity inside the self-pulsing domain. At a fixed value of the ion pair concentration, the increase in pumping gives rise to pulses of a higher repetition rate and close to the bifurcation point the intensity becomes sinusoidal. Besides, pulse amplitude reaches a maximum approximately in the middle of the selfpulsing domain.

\subsection{The two-mode erbium-doped fiber laser}

A further step in the study of the laser dynamics considers a dual wavelength operation at $1.550 \mu \mathrm{m}$ and $1.556 \mu \mathrm{m}$, treated as a two coupled laser modes (Sanchezet al., 1995).

The nonlinear dynamics of a two-mode fiber laser is explained in terms of a classical twomode laser model and two additional equations for the ion-pair quantum states.

The laser is described through six coupled differential equations. There are analyzed the steady-states, their dependence on the laser parameters, and the onset of the self-pulsing and chaotic states is pursued.

The two modes of the laser are coupled via a cross-saturation parameter $(\beta)$. The system is described through the set of coupled equations:

$$
\begin{aligned}
& \dot{d}_{1}=\Lambda-a_{2}\left(1+d_{1}\right)-2 d_{1}\left(I_{1}+\beta I_{2}\right), \\
& \dot{d}_{2}=\gamma \Lambda-a_{2}\left(1+d_{2}\right)-2 d_{2}\left(\beta I_{1}+I_{2}\right),
\end{aligned}
$$




$$
\begin{gathered}
\dot{d}_{+}=a_{2}\left(1-d_{+}\right)-\left(a_{22} / 2\right)\left(d_{+}+d_{-}\right)+y\left(2-3 d_{+}\right)\left(I_{1}+I_{2}\right), \\
\dot{d}_{-}=\Lambda-a_{2}\left(1-d_{+}\right)-\left(a_{22} / 2\right)\left(d_{+}+d_{-}\right)-y d_{-}\left(I_{1}+I_{2}\right), \\
\dot{I}_{1}=-I_{1}+A(1-2 x)\left(d_{1}+\beta d_{2}\right) I_{1}+A x y d_{-} I_{1}, \\
\dot{I}_{2}=-I_{2}+A(1-2 x)\left(\beta d_{1}+d_{2}\right) I_{2}+A x y d_{-} I_{2} .
\end{gathered}
$$

In the above, $I_{1,2}$ are the normalized intensities and $d_{1,2}$ are the normalized population inversions of the two modes. The supplementary parameter $\gamma$ takes into account the anisotropy in pumping for the two modes.

System (2) is to be investigated for typical parameters: $\tau_{L}=200 \mathrm{~ns}, \tau_{2}=10 \mathrm{~ms}, \tau_{22}=2 \mu \mathrm{s}$, $N_{0}=5 \times 10^{18} \mathrm{~cm}^{-3}, \sigma_{\mathrm{L}}=1.6 \times 10^{-10} \mathrm{~cm}^{3} \mathrm{~s}^{-1}, y=0.2, \beta=0.5$ and $\gamma=0.9$.

There are three types of interesting stationary states: $I_{1}=I_{2}=0$ (laser below threshold), $\left(I_{1}>0, I_{2}=0\right)$, i.e., the single-mode laser, and $I_{1,2}>0$ (the two-mode state). The complete determination requires the solving of a fourth-order polynomial equation for intensity in the single-mode case, and a third-order polynomial equation in the two-mode case. The treatment of the steady-state stability is performed through the linearization of the system (2) around the steady-states and searching of the eigenvalues of a six-order matrix ( Crawford,1991; Abarbanel,1996; Ştefănescu,2002). The results are showed in Fig 13(a) for the parameters above and a range of ion-pair concentration in accordance with all practical cases. Here, $\Lambda_{\text {th }}^{(1)}$ denotes the pumping parameter at the laser threshold and $\Lambda_{\text {th }}^{(2)}$ is the pumping parameter at the switching of the weak mode (mode 2 , here). We focus here on the two-mode operation. The pumping strength is usually expressed in the form $r=\Lambda / \Lambda_{\mathrm{th}}^{(1)}$. The steady-state intensities versus the pumping parameter are presented in figure 14 . The straight-line dependence is followed for a large range of pumping strengths.

The way to a stable steady-state is always through relaxation oscillations (Fig. 15).

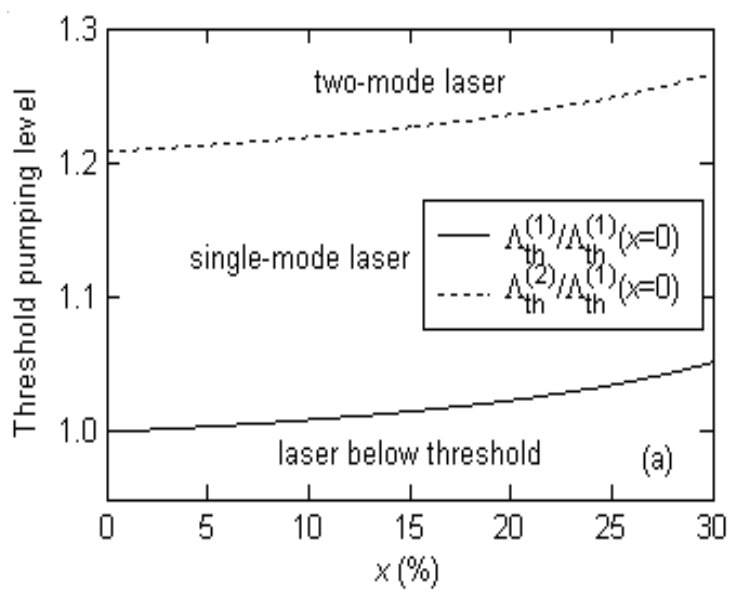

Fig. 13. Threshold pumping parameter for the laser action and two-mode states vs. ion pair percentage 


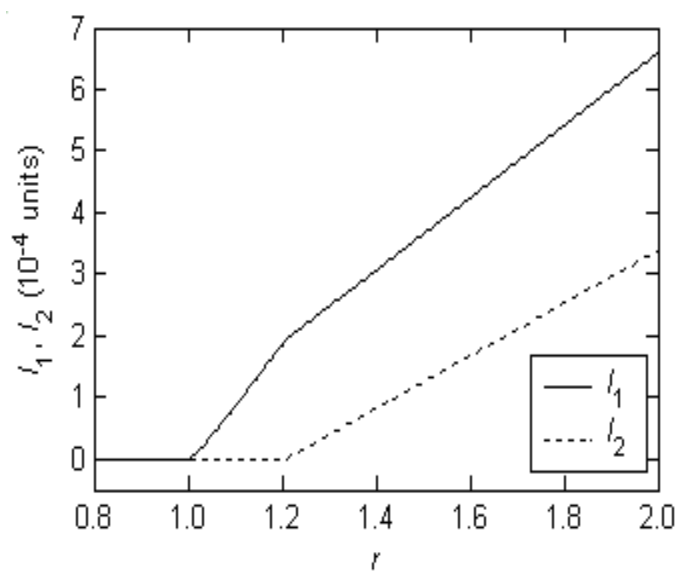

Fig. 14. Steady-state intensities of the two-mode laser versus the pumping parameter, $x=0.1$
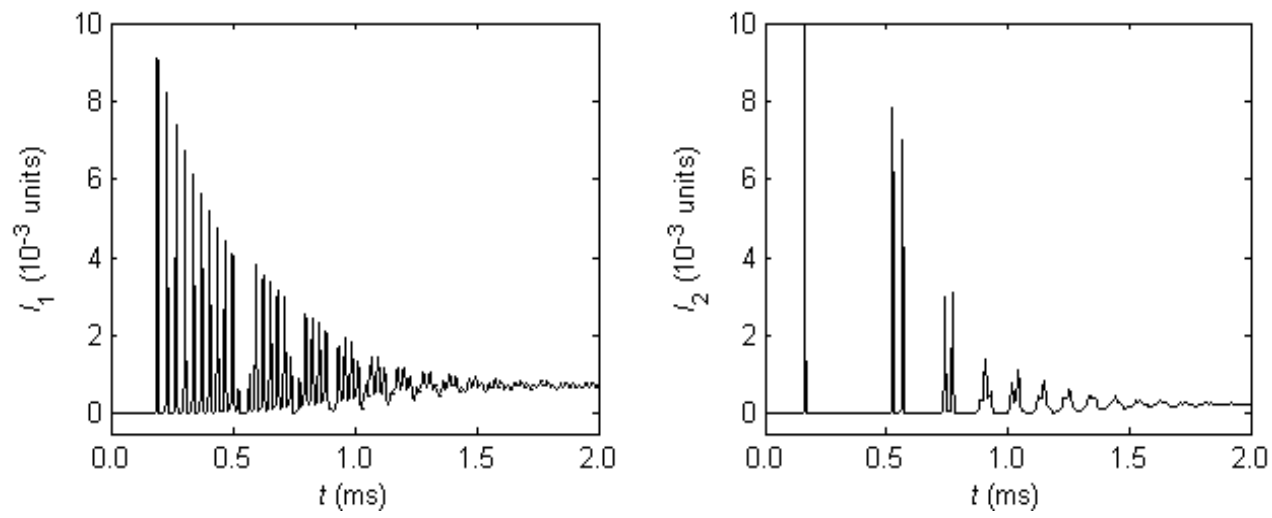

Fig. 15. (a) Transitory regime to a two-mode stationary state. $x=0.03$ and $r=1.5$.

A thorough investigation of the laser dynamics in the domain where both modes are active seems to be a hard task. Instead, we try to find out the basics of this. For sufficiently low doping level, the asymptotic dynamics is a steady-state whatever the pumping parameter.

For larger values of $x$, there is a range of self-pulsation dynamics. In Fig.16(a) this is showed by means of a bifurcation diagram, i.e., the maxima of one mode intensity is plotted against the pumping parameter. The dynamics in Fig. 16(a) has also been encountered in the case of the one-mode model (Sanchez et al., 1993) and the change from a stationary stable state to a self-pulsing one at $r \approx 1.8$ is a Hopf bifurcation (Crawford, 1991).

There exists a region of two maximum intensities, i.e., a period doubling. Temporal dynamics in such a case (Fig. 17) exhibits antiphase dynamics of the two laser modes. 
A small increase of the ion-pair concentration to the value of 7\% [Fig.16(c)] leads to the appearance of a window of a tripled period [Fig. (17c)]. Further increase of the ion-pair concentrations leads to a more complicated bifurcation diagram, including regions with multiple stable states (generalized bistability) and routes to chaotic dynamics such as the period-doubling route or a quasi-periodic one.
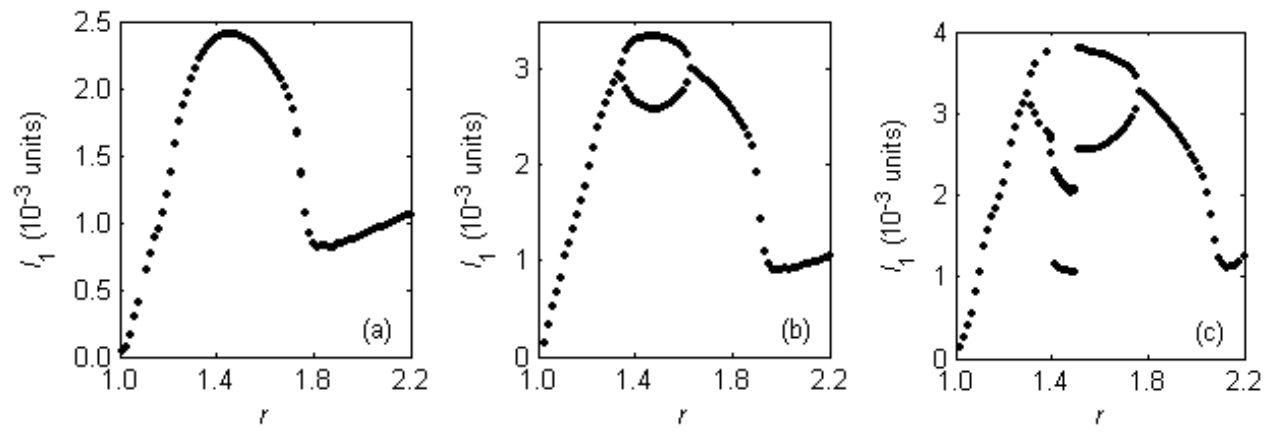

Fig. 16. Bifurcation diagram of the strong mode intensity for relatively small ion-pair percentages: (a) $x=0.065$, (b) $x=0.068$, and (c) $x=0.070$. Figure 16(b) shows the maxima of intensity $I_{1}$ at a slightly larger ion-pair concentration.
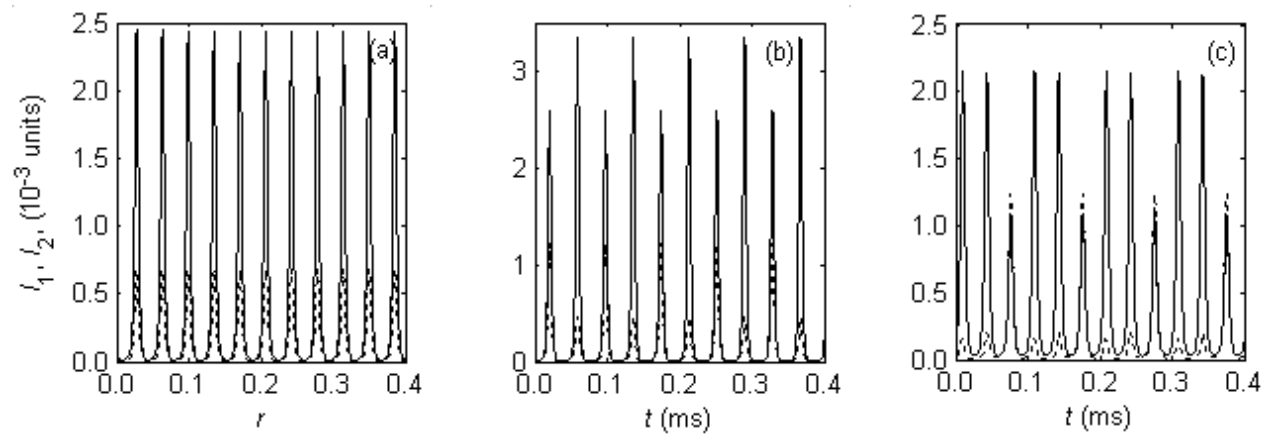

Fig. 17. Long-term temporal evolution of the laser for $r=1.45$ and ion-pair concentrations in Fig. 6: (a) $x=0.065$, (b) $x=0.068$, and (c) $x=0.070$. Solid line is used for the strong mode $\left(I_{1}\right)$ and dotted line for the weak mode $\left(I_{2}\right)$.

\section{Conclusions}

The developed numerical models concerning the characterization and operation of the EDFA systems and also of the laser systems, both of the "crystal type" or "fiber type" realized in $\mathrm{Er}^{3+}$ doped media and the obtained results are consistent with the existing data in 
the literature. Our results put into evidence the existence of the new situations which are important for the optimization of the functioning conditions for this kind of devices.

That was due to the valences of the computer experiment method which make possible a complex study taking into account parameters intercorrelations by simulating experimental conditions, as have been shown.

The erbium ion pairs in a laser fiber can explain the experimentally observed nonlinear dynamics of the system, apart from the intrinsic nonlinearity of a multiple-mode laser. The existence of the erbium ion pairs introduces a supplementary nonlinearity with a saturable absorber action (Sanchez et al.,1995). In the single-mode laser description, as well as in the two-mode laser the ion pairs are responsible for an increase of the laser threshold, a decrease of the laser gain, and self-pulsations. Depending on the laser pumping level, the laser self-pulsations range from an oscillatory form to a well-defined pulse-shape. The nonlinear dynamics is present even close to the laser threshold and does not requires large pumping levels.

The nonlinear dynamics can be avoided by a choice of sufficiently low-doped fibers, such that the average distance between ions is large enough and the interaction between ions is small. It is clear that the limitation to sufficiently low-doped fibers is performed at the expense of using longer fibers.

\section{References}

Abarbanel, H. (1996). Analysis of observed chaotic data, Springer-Verlag, Berlin.

Agrawal, G.P. (1995). Nonlinear fiber optics, Academic Press, San Diego.

Agrawal, G.P. (1997). Optic Communication Systems, A Wiley - Interscience Publication, J. Wiley and Sons, Inc.,New York.

Baker, G. L. \& Gollub, J. P. (1990). , Chaotic dynamics. An introduction, Cambridge University Press, Cambridge.

Crawford, J. D. (1991). Introduction to bifurcation theory, Rev. Mod. Phys. 63, 991-1037, (1991).

Desurvire, E. (1995). Erbium - Dopped Fiber Amplifier, J. Wiley and Sons, Inc., New York.

Le Flohic, M.; Francois, P.L.; Allain, J. Y. ; Sanchez, F. \& Stephan, G. (1991). Dynamics of the transient buildup of emission in $\mathrm{Nd}^{3+}$ doped fiber lasers, IEEE J. Quantum Electron. 27, 1910-1921, (1991).

Maciuc, F. C.; Stere, C. I \& Sterian, A.R. (2001). Rate equations for an Erbium laser system, a numerical approach, Proceedings of SPIE, Vol. 4430, 136 - 146, The Sixth Conference on Optics ROMOPTO 2000, Bucharest.

Maciuc, F. C.; Stere, C. I \& Sterian, A.R. (2001). Time evolution and multiple parameters variations in a time dependent numerical model applied for $\mathrm{Er}^{+3}$ laser system, Proceedings of SPIE, Vol. 4394, 84 - 89, Laser Physics and Application 10 International School an Quantum Electronics (2001), Varna.

Ninulescu, V.; Sterian, A. R. \& Sterian, P. (2006). Dynamics of a two-mode erbium-doped fiber laser, Proceedings of SPIE, Vol. 6344, 63440 Q1 - 63440Q6, ALT-05, (Editors: Ivan A. Shcherbakov ş.a.), June (2006), Tianjin. 
Ninulescu, V. \& Sterian, A. R. (2005). Dynamics of a Two-Level Medium Under the Action of Short Optical Pulses, Lecture Notes in Computer Science, LNCS 3482, 635-642, Gervasi, O.et al. (Eds), Springer - Verlag, (2005), Berlin, Heidelberg.

Petrescu, A.; Sterian, A. R. \& Sterian, P.E. (2007). Solitons propagation in optical fibers: computer experiments for students training, Proceedings of Computational Science and Its Applications, ICCSA 2007, Pt 1, 4705450-461, Editor(s): Gervasi, O; Gavrilova M.L., (2007).

Pollnau, M.; Graf, Th.; Balmer, J. E.; Lüthy, W. \& Weber H. P. (1994). Explanation of the cw operation of the $\mathrm{Er}^{3+} 3-\mu \mathrm{m}$ crystal laser, Phys. Rev. A 49 (5), 3990-3996, (1994).

Pollnau, M.; Spring, R.; Ghisler, Ch.; Wittwer, S.; Luthy, W. \& Weber, H.P. (1996). Efficiency of Erbium 3- $\mu \mathrm{m}$ crystal and fiber lasers.IEEEJ Quantum Electronics, Vol.32, No. 4, April (1996).

Press, W. H.; Teukolsky, S.A.; Vetterling, W.T. \& Flannery, B.P. (1992). Numerical Recipients in C: The art of Scientific Compunting, Second Edition, Cambridge University Press, Cambridge.

Sanchez, F.; Le Flohic, M. ; Stephan, G. M. ; Le Boudec, P.\& Francois, P.L. (1995). Quasi periodic route to chaos in erbium-doped fiber laser, IEEE J. Quantum Electron. 31, 481- 488, (1995).

Sanchez, F.; Le Boudec, P.; Francois, P.L. \& Stephan, G. (1993). Effects of ion pairs on the dynamics of erbium - doped fiber lasers, Phys. Rev. A 48, 2220-2229. (1993)

Shalibeik, H. (2007). Rare-Earth-Doped Fiber Lasers and Amplifiers. Cuvillier Verlag, 978-386727-467-8, Gottingen.

Stefanescu, E.N.; Sterian, A. R. \& Sterian, P. E. (2005). Study of the fermion system coupled by electric dipol interaction with the free electromagnetic field, Proceedings of SPIE, Vol. 5850, 160-165, Advanced Laser Tehnologies 2004, edited by Anna Giardini, Vitali I. Konov, Vladimir I. Pustavoy, (2005), Rome.

Stefanescu, E.N.; Sterian, P. E. \& Sterian, A. R. (2000). Fundamental Interactions in Dissipative Quantum Systems, Hyperion University Scientific Bulletin, Vol.1, No 1, 87-92, (2000).

Stefanescu, E.N.; Sterian, P. E. \& Sterian, A. R. (2002). The Lindblad dynamics of a Fermi system in a particle dissipative environment, Proceedings of SPIE, Vol. 5147, 160168, ALT 02, (2002), Adelboden.

Sterian, A. R. \& Ninulescu, V. (2005). Nonlinear Phenomena in Erbium-Doped Lasers, Lecture Notes in Computer Science, LNCS 3482, 643-650, Gervasi, O.et al. (Eds), Springer - Verlag, (2005), Berlin, Heidelberg.

Sterian, A.R. (2006). Amplificatoare optice, Editura Printech, ISBN 978-973-718-434-4, Bucuresti.

Sterian, A.R. \& Maciuc, F. C. (2003). Numerical model of an EDFA based on rate equations, Proceedings of SPIE, Vol.

5226, 74-78, Laser Physics and Application 12th International School an Quantum Electronics (2003), Varna.

Sterian, A.R. (2008). Mecanica cuantică, Omnia Univ. S.A.S.T., (ISBN 978-973-134-042-5, 978973-134-040-1), Braşov. 
Sterian, A.R. (2007). Computer modeling of the coherent optical amplifier and laser systems, Proceedings of Computational Science and Its Applications, ICCSA 2007, Pt 1, 4705436449, Editor(s): Gervasi, O; Gavrilova M.L., (2007). 


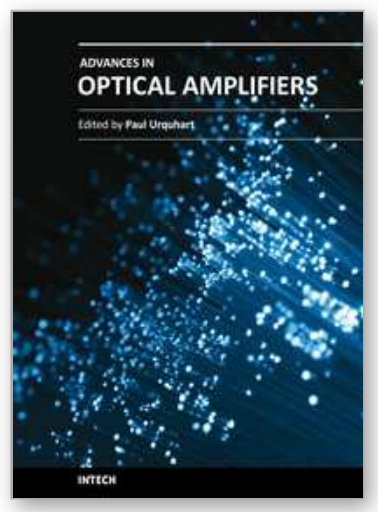

\author{
Advances in Optical Amplifiers \\ Edited by Prof. Paul Urquhart
}

ISBN 978-953-307-186-2

Hard cover, 436 pages

Publisher InTech

Published online 14, February, 2011

Published in print edition February, 2011

Optical amplifiers play a central role in all categories of fibre communications systems and networks. By compensating for the losses exerted by the transmission medium and the components through which the signals pass, they reduce the need for expensive and slow optical-electrical-optical conversion. The photonic gain media, which are normally based on glass- or semiconductor-based waveguides, can amplify many high speed wavelength division multiplexed channels simultaneously. Recent research has also concentrated on wavelength conversion, switching, demultiplexing in the time domain and other enhanced functions. Advances in Optical Amplifiers presents up to date results on amplifier performance, along with explanations of their relevance, from leading researchers in the field. Its chapters cover amplifiers based on rare earth doped fibres and waveguides, stimulated Raman scattering, nonlinear parametric processes and semiconductor media. Wavelength conversion and other enhanced signal processing functions are also considered in depth. This book is targeted at research, development and design engineers from teams in manufacturing industry, academia and telecommunications service operators.

\title{
How to reference
}

In order to correctly reference this scholarly work, feel free to copy and paste the following:

Sterian Andreea Rodica (2011). Coherent Radiation Generation and Amplification in Erbium Doped Systems, Advances in Optical Amplifiers, Prof. Paul Urquhart (Ed.), ISBN: 978-953-307-186-2, InTech, Available from: http://www.intechopen.com/books/advances-in-optical-amplifiers/coherent-radiation-generation-andamplification-in-erbium-doped-systems

\section{INTECH}

open science | open minds

\section{InTech Europe}

University Campus STeP Ri

Slavka Krautzeka 83/A

51000 Rijeka, Croatia

Phone: +385 (51) 770447

Fax: +385 (51) 686166

www.intechopen.com

\section{InTech China}

Unit 405, Office Block, Hotel Equatorial Shanghai

No.65, Yan An Road (West), Shanghai, 200040, China 中国上海市延安西路65号上海国际贵都大饭店办公楼 405 单元

Phone: +86-21-62489820

Fax: +86-21-62489821 
(C) 2011 The Author(s). Licensee IntechOpen. This chapter is distributed under the terms of the Creative Commons Attribution-NonCommercialShareAlike-3.0 License, which permits use, distribution and reproduction for non-commercial purposes, provided the original is properly cited and derivative works building on this content are distributed under the same license. 